\title{
Enduring epigenetic landmarks define the cancer microenvironment
}

Ruth Pidsley, 1,2,17 Mitchell G. Lawrence, ${ }^{3,4,17}$ Elena Zotenko, ${ }^{1,2}$ Birunthi Niranjan, $^{3}$ Aaron Statham, ${ }^{1}$ Jenny Song, ${ }^{1}$ Roman M. Chabanon, ${ }^{1}$ Wenjia Qu, ${ }^{1}$ Hong Wang, ${ }^{3}$ Michelle Richards, ${ }^{3}$ Shalima S. Nair, ${ }^{1,2}$ Nicola J. Armstrong, ${ }^{1,5}$ Hieu T. Nim, ${ }^{3,6,7}$ Melissa Papargiris, ${ }^{3}$ Preetika Balanathan, ${ }^{3}$ Hugh French, ${ }^{1}$ Timothy Peters, ${ }^{1}$ Sam Norden, ${ }^{8}$ Andrew Ryan, ${ }^{8}$ John Pedersen, 3 ,8 James Kench, ${ }^{9,10}$ Roger J. Daly, ${ }^{11}$ Lisa G. Horvath, ${ }^{9,12,13}$ Phillip Stricker, ${ }^{9,14}{ }^{\text {Mark Frydenberg, }}{ }^{3}$ Renea A. Taylor, ${ }^{4,15}$ Clare Stirzaker, ${ }^{1,2}$ Gail P. Risbridger, ${ }^{3,4,16,17}$ and Susan J. Clark 1,2,17

${ }^{1}$ Epigenetics Research Laboratory, Genomics and Epigenetics Division, Garvan Institute of Medical Research, Darlinghurst, Sydney, New South Wales 2010, Australia; ${ }^{2}$ St. Vincent's Clinical School, UNSW Sydney, New South Wales 2052, Australia; ${ }^{3}$ Prostate Research Group, Cancer Program -Biomedicine Discovery Institute, Department of Anatomy and Developmental Biology, Monash Partners Comprehensive Cancer Consortium, Monash University, Clayton, Victoria 3800, Australia; ${ }^{4}$ Prostate Cancer Translational Research Program, Cancer Research Division, Peter MacCallum Cancer Centre, Melbourne, Victoria 3000, Australia; ${ }^{5}$ Mathematics and Statistics, Murdoch University, Perth, Western Australia 6150, Australia; ${ }^{6}$ Faculty of Information Technology, Monash University, Clayton, Victoria 3800, Australia; ${ }^{7}$ Australian Regenerative Medicine Institute, Monash University, Clayton, Victoria 3800, Australia;

${ }^{8}$ Tissupath Pathology, Mount Waverley, Victoria 3149, Australia; ${ }^{9}$ Cancer Research Division, Garvan Institute of Medical Research/ The Kinghorn Cancer Centre, Darlinghurst, New South Wales 2010, Australia; ${ }^{10}$ Department of Tissue Pathology and Diagnostic Oncology, Royal Prince Alfred Hospital, Camperdown, Sydney, New South Wales 2050, Australia; ${ }^{11}$ Signalling Network Laboratory, Department of Biochemistry and Molecular Biology, Biomedicine Discovery Institute, Monash Partners Comprehensive Cancer Consortium, Monash University, Clayton, Victoria 3800, Australia; ${ }^{12}$ Chris O'Brien Lifehouse, Missenden Road, Camperdown, New South Wales 2050, Australia; ${ }^{13}$ University of Sydney, Sydney, New South Wales 2050, Australia; ${ }^{14}$ Department of Urology, St. Vincent's Prostate Cancer Centre, Sydney, New South Wales 2050, Australia; ${ }^{15}$ Prostate Research Group, Cancer ProgramBiomedicine Discovery Institute Department of Physiology, Monash Partners Comprehensive Cancer Consortium, Monash University, Clayton, Melbourne, Victoria 3800, Australia; ${ }^{16}$ Sir Peter MacCallum Department of Oncology, The University of Melbourne, Victoria 3010, Australia

\begin{abstract}
The growth and progression of solid tumors involves dynamic cross-talk between cancer epithelium and the surrounding microenvironment. To date, molecular profiling has largely been restricted to the epithelial component of tumors; therefore, features underpinning the persistent protumorigenic phenotype of the tumor microenvironment are unknown. Using whole-genome bisulfite sequencing, we show for the first time that cancer-associated fibroblasts (CAFs) from localized prostate cancer display remarkably distinct and enduring genome-wide changes in DNA methylation, significantly at enhancers and promoters, compared to nonmalignant prostate fibroblasts (NPFs). Differentially methylated regions associated with changes in gene expression have cancer-related functions and accurately distinguish CAFs from NPFs. Remarkably, a subset of changes is shared with prostate cancer epithelial cells, revealing the new concept of tumor-specific epigenome modifications in the tumor and its microenvironment. The distinct methylome of CAFs provides a novel epigenetic hallmark of the cancer microenvironment and promises new biomarkers to improve interpretation of diagnostic samples.
\end{abstract}

[Supplemental material is available for this article.]

Most studies of the genome and epigenome landscape of tumors focus on the aberrations in epithelial cancer cells. However, solid

\footnotetext{
${ }^{17}$ These authors contributed equally to this work. Corresponding authors: s.clark@garvan.org.au, gail.risbridger@ monash.edu

Article published online before print. Article, supplemental material, and publication date are at http://www.genome.org/cgi/doi/10.1101/gr.229070.117. Freely available online through the Genome Research Open Access option.
}

tumors are embedded in an interactive and heterogenous cellular microenvironment (Hanahan and Weinberg 2011), which includes a predominant population of cancer-associated fibroblasts (CAFs) that can also display cellular heterogeneity (Franco et al. 2011; Kiskowski et al. 2011). Early pathology studies (Seemayer

(C) 2018 Pidsley et al. This article, published in Genome Research, is available under a Creative Commons License (Attribution-NonCommercial 4.0 International), as described at http://creativecommons.org/licenses/by-nc/4.0/. 
et al. 1979) and subsequent functional experiments (Olumi et al. 1999; Bhowmick et al. 2004) identified that prostatic CAFs promote tumor development and progression. CAFs also enhance the migration and proliferation of prostate cancer cells in vitro (Ao et al. 2007; Paland et al. 2009; Clark et al. 2013). The differences between CAFs and NPFs are known to be permanent, as CAFs retain their protumorigenic phenotype and characteristic gene expression signature in primary cell culture without epithelial cells (Zhao et al. 2007).

It remains unknown how these enduring phenotypic changes in CAFs are encoded at the molecular level. Initial studies suggested that CAFs acquire genetic aberrations, but no recurrent genome alterations were identified in subsequent reports (Qiu et al. 2008; Ashida et al. 2012; Bianchi-Frias et al. 2016). Here we ask if genome-wide differences in the epigenome underpin the persistent phenotypic differences between CAFs and NPFs. Earlier studies using low-resolution techniques found examples of DNA methylation changes in CAFs or tumor stroma from various cancer types ( $\mathrm{Hu}$ et al. 2005; Fiegl et al. 2006; Hanson et al. 2006; Rodriguez-Canales et al. 2007; Dawsey et al. 2008; Jiang et al. 2008; Banerjee et al. 2014; Vizoso et al. 2015). However, the full epigenome landscape of CAFs remains uncharted, so the precise epigenetic changes and their contribution to altered transcriptional patterns is unknown.

To explore the epigenetic and functional alterations that define CAFs, we used whole-genome bisulfite sequencing (WGBS) to profile the DNA methylome of CAFs and matched NPFs from patients with localized prostate cancer. In parallel, we used single-nucleotide polymorphism (SNP) arrays to assess genomic integrity, RNA-seq to measure mRNA abundance, and DNA methylation arrays and targeted bisulfite sequencing to determine the technical and biological reproducibility of changes in DNA methylation between patients.

\section{Results}

\section{CAFs from a spectrum of localized prostate cancers are protumorigenic}

To investigate functional and molecular changes that arise in the prostate cancer microenvironment of different patients, we used primary cells from 17 men undergoing a radical prostatectomy. The cohort is typical of the diverse spectrum of localized sporadic prostate cancers treated with curative intent, including moderateto high-grade disease (Supplemental Table S1). To isolate and purify CAFs and NPFs, we first established primary cultures, which are likely heterogeneous populations of fibroblast subgroups, from digested tumor tissue and matched nonmalignant tissue (Fig. 1A; Supplemental Table S1). All patient specimens were carefully validated using pathology (Fig. 1B), and cultures of CAFs and NPFs were shown to have typical fibroblast morphology and expressed stromal (vimentin), but not epithelial markers (cytokeratin 8/18, high molecular weight cytokeratin) (Supplemental Table S1).

Next, we verified the phenotypic and functional differences between patient-matched sets of fibroblasts. CAFs and NPFs differentially expressed several markers previously used to differentiate between CAFs and NPFs, including SFPR1 (Supplemental Fig. S1A; Supplemental Table S1; Joesting et al. 2005; Franco et al. 2011; Kiskowski et al. 2011; Orr et al. 2012). We found that CAFs were protumorigenic in classical in vivo tissue recombination as-

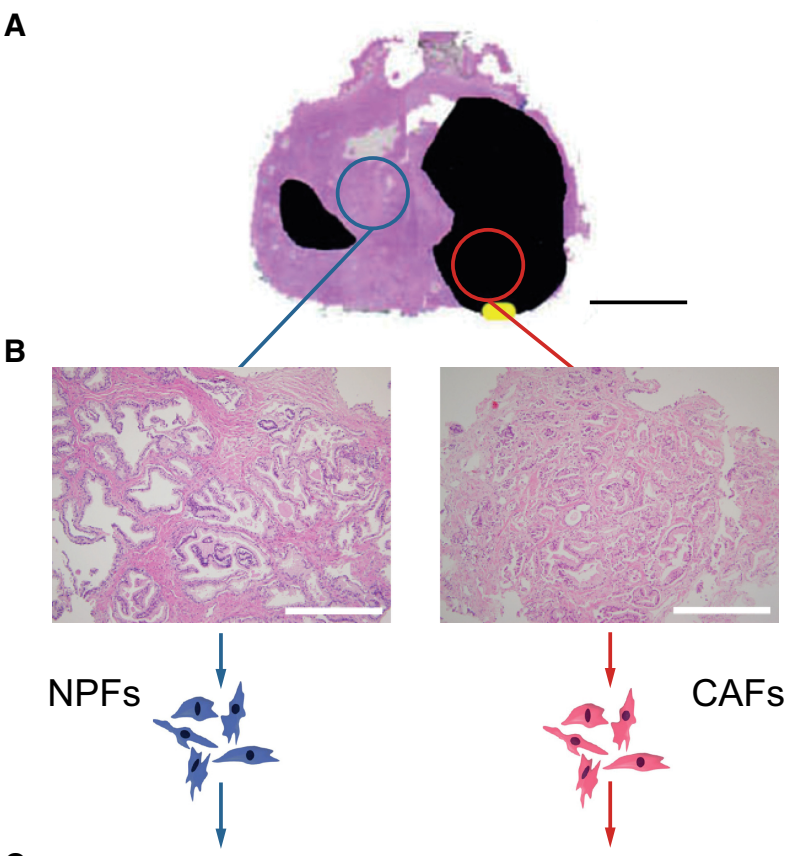

C
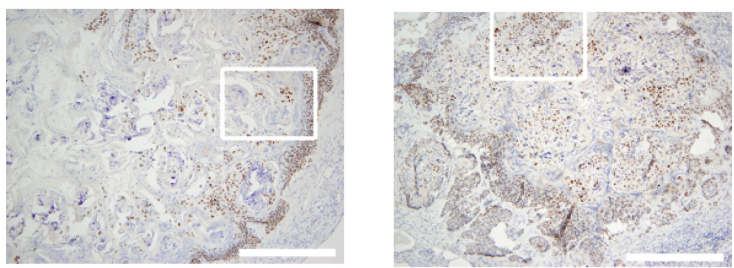

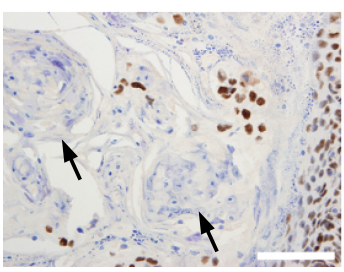

$\mathrm{BPH}-1+\mathrm{NPF}$

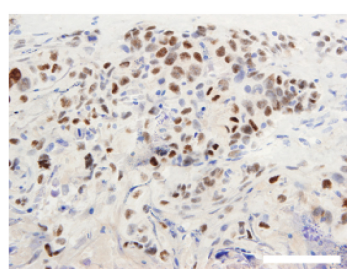

$\mathrm{BPH}-1+\mathrm{CAF}$
Figure 1. Isolation and validation of matched CAFs and NPFs from patient tissue. Representative data from patient 1. (A) Matched nonmalignant (blue) and cancer (red) tissue were dissected from the same radical prostatectomy specimen and used to establish primary cultures of CAFs and NPFs. (Scale) $2 \mathrm{~cm}$. (B) Hematoxylin and eosin staining confirmed that NPFs were from a nonmalignant region and that CAFs were from a region of Gleason 3+4 cancer. (Scales) $500 \mu \mathrm{m}$. (C) Grafts from tissue recombination assays with BPH-1 epithelial cells stained for SV40 T antigen (brown) and nuclei counterstained with hematoxylin (blue). BPH-1 cells combined with NPFs formed well-differentiated structures, evident from organized layers of epithelial cells with abundant keratinization (arrows). In contrast, BPH-1 cells combined with CAFs formed poorly differentiated structures, consistent with tumorigenesis, apparent from the disorganized clusters of epithelial cells and limited keratinization. White boxes indicate the areas shown in higher magnification. (Scales) $500 \mu \mathrm{m}$ for low magnification images and $100 \mu \mathrm{m}$ for high magnification images.

says (Fig. 1C; Supplemental Table S1) and in a novel in vitro coculture assay (Supplemental Table S1; Clark et al. 2013). Collectively, these data confirmed that the primary cells exhibit the defining functional features of CAFs and NPFs, making this one of the largest and most rigorously profiled cohorts of patient-matched fibroblasts for prostate cancer.

\section{Genome Research}

www.genome.org 
Prostate cancer fibroblasts show discrete changes in DNA methylation but no genetic copy number changes

To determine if there were genetic copy number alterations, we assayed the low passage (P2-6) patient-matched fibroblasts (patients 1-4) on the Illumina Infinium Omni2.5-8 BeadChip genotyping platform and found no evidence of large-scale genomic aberrations within any NPF-CAF patient pair (Fig. 2A; Supplemental Fig. S1B). Next, we used WGBS to comprehensively analyze the DNA methylation profiles of the same samples. We obtained $>7 \times$ coverage for each sample and efficient bisulfite conversion (98.5\%-99.7\%). For cross-platform validation, genome-wide methylation was measured using the Illumina Infinium HumanMethylation450 BeadChip (450K) and showed excellent agreement between replicates (Pearson's $r=0.88-0.90, P<2.2 \times 10^{-16}$ ) (Supplemental Fig. $\mathrm{S} 1 \mathrm{C})$. Overall, we found that the genome-wide methylation profiles were highly correlated between NPFs and CAFs (Pearson's $r=0.90$, $P<2.2 \times 10^{-16}$ ) (Fig. 2B), particularly compared to other related cell types (Supplemental Fig. S1D). Both CAFs and NPFs displayed typical bimodal distributions of CpG methylation (Fig. 2B) and similar
A

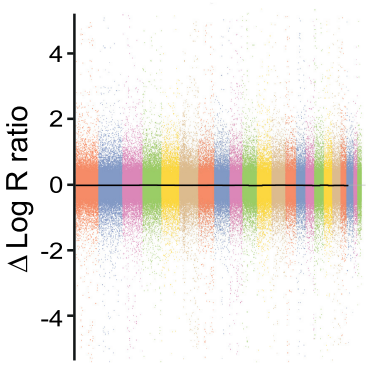

B

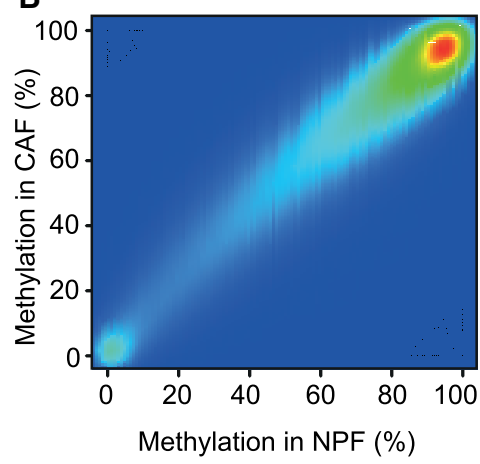

C

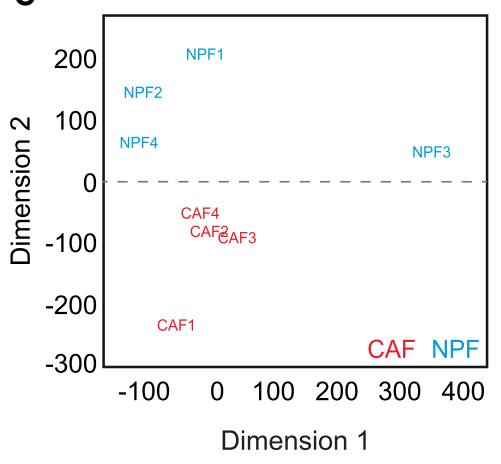

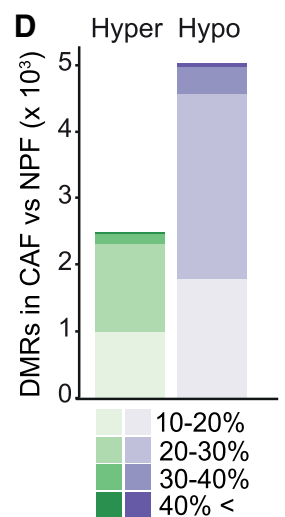

$E$

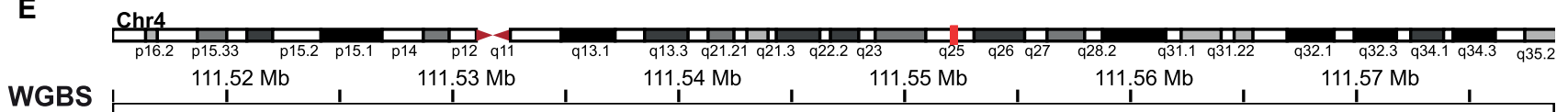

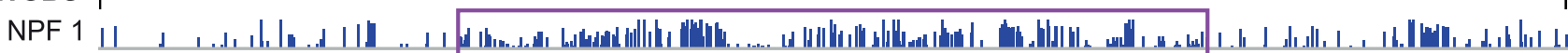

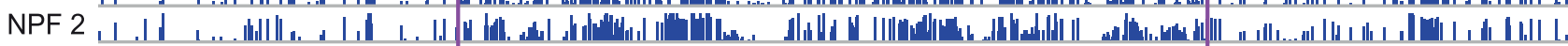

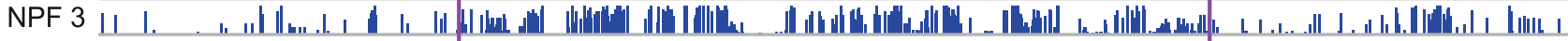

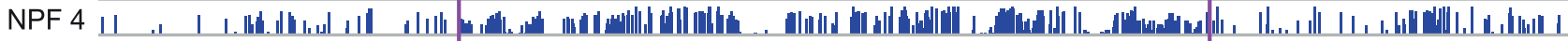

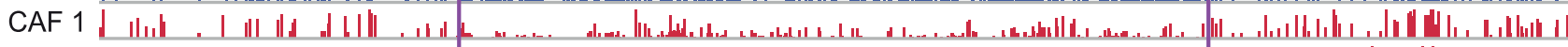

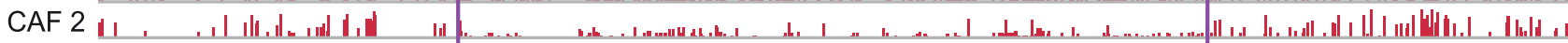

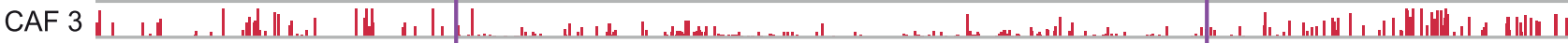

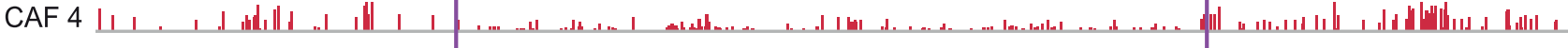

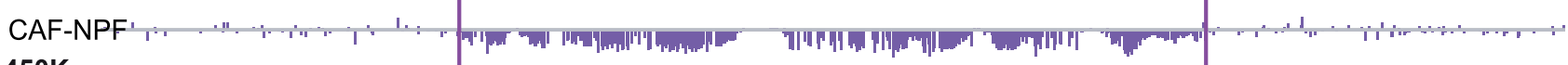

\section{K}

Probes

NPF 1

NPF 2

NPF 3

NPF 4

CAF 1

CAF 2

CAF 3

CAF 4

CAF-NPF

PITX2

\begin{tabular}{|c|c|c|c|c|}
\hline | || |||||||| || || || || || || | || || || |||||| || || ||| |||| || || & 11 & ||||||| ||||| || || || ||||| & |IIII| || ||| || & \\
\hline $\mid$ & 11 & hill llit. I. I. &. $.11 \quad$ IIII & \\
\hline 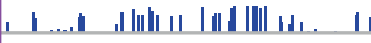 & Il & 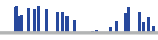 & .ll ull I. & \\
\hline $\mid$ & ॥ & 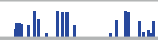 & . Il ill. & \\
\hline $\mid$ & Il & 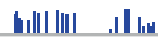 & ...ll ulı & \\
\hline $11,1, \ldots 1$, & & 1 ... .... & $\ldots$ & \\
\hline 1. & & & & \\
\hline & & d & & \\
\hline .6 $\quad$ L......... & .. & C.IL & & \\
\hline 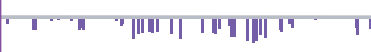 & $\Pi$ & 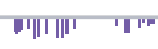 & 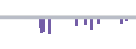 & \\
\hline
\end{tabular}

Figure 2. CAFs and NPFs have similar global methylation profiles but discrete methylomic differences. ( $A$ ) Representative SNP array data (patient 1 ) showing no large-scale genomic aberrations. (B) High correlation $\left(r=0.90, P<2.2 \times 10^{-16}\right)$ of WGBS DNA methylation levels between CAFs and NPFs ( $n=4$ pairs) indicates no global perturbations between cell types. (C) MDS plot of the $10 \%$ most variably methylated regions shows clear separation of CAFs and NPFs. $(D)$ The number of hypermethylated (green) and hypomethylated (purple) DMRs in CAFs according to the percentage change in DNA methylation. (E) WBGS and 450K data for PITX2 for each NPF (blue) and CAF (red). The average difference in DNA methylation in CAFs compared to NPFs is shown in purple. The height of each vertical line represents the percentage of DNA methylation at each $\mathrm{CpG}$ site. The locations of $450 \mathrm{~K}$ probes are shown in gray. The purple box highlights a large region of hypomethylation measured on both platforms. 
overall levels of methylation across CpG islands, shores, and nonCpG islands (Supplemental Fig. S1E). Notably, we found no evidence of the global hypomethylation that typically occurs in tumor epithelial cells (Supplemental Fig. S1E,F) and as previously reported for CAFs from gastric and non-small cell lung cancers (Jiang et al. 2008; Vizoso et al. 2015).

Despite the lack of global differences in methylation between CAFs and NPFs, multidimensional scaling analysis of the most variably methylated CpG sites showed clear separation by cell type, indicating regions of differential methylation between CAFs and NPFs (Fig. 2C). Using bsseq analysis (Hansen et al. 2012), we identified 7534 distinct differentially methylated regions (DMRs) between patient-matched CAFs and NPFs (absolute methylation difference $>10 \%$, with a minimum of three CpGs sites per region) (Supplemental Table S2). We defined hypomethylated and hypermethylated DMRs as discrete regions with, respectively, decreased or increased methylation in CAFs compared to NPFs. We found that CAFs have more hypomethylated DMRs $(n=5038,2.2 \mathrm{Mb}$ of the genome) compared to hypermethylated DMRs ( $n=2496$; $1.1 \mathrm{Mb}$ of the genome) (Fig. 2D), although hypomethylated DMRs tend to be slightly smaller (460 vs. 428 bp; MannWhitney $U$ test $P=4.8 \times 10^{-7}$ ) (Supplemental Fig. S2A). When we grouped DMRs by the level of methylation difference (Fig. 2D), we observed that regions with greater differences are significantly larger in size (Spearman's correlation hypermethylated $\rho=0.37$, $P<2.2 \times 10^{-16}$, and hypomethylated $\rho=-0.35, P<2.2 \times 10^{-16}$ ) (Supplemental Fig. S2B). Notably, the regions with the greatest methylation differences also occur at sites with either low (hyper) or high (hypo) methylation in NPFs (Supplemental Fig. S2C). We also found significant cross-platform validation of DMRs for each patient (Supplemental Fig. S2D,E). For example, an $\sim 400-\mathrm{kb}$ region spanning the gene body of PITX2 showed extensive hypomethylation in all four CAFs compared to patient-matched NPFs in both WGBS and 450K data sets (Fig. 2E).

\section{Differential methylation in CAFs occurs at regulatory regions}

To examine the potential functional importance of DMRs in CAFs, we determined their location relative to different genomic features (Fig. 3A,B). We found that most DMRs are outside CpG islands, although the proportion of DMRs overlapping CpG islands does increase with the degree of methylation change between CAFs and NPFs (Fig. 3B). Relative to annotated protein-coding genes, $45 \%$ of hypermethylated and 39\% of hypomethylated DMRs are in gene bodies (Fig. 3B). Hypomethylated DMRs are more common in gene promoters (33\% hypomethylated vs. $21 \%$ hypermethylated) and are located closer to transcription start sites (TSSs) compared to hypermethylated DMRs (median distance to TSS hypomethylated DMRs $=9343$ bp vs. hypermethylated DMRs $=15,726$ bp, Mann-Whitney $U$ test, $P=2.6 \times 10^{-18}$ ) (Supplemental Fig. S2F).

To further examine the association between DMRs and regulatory elements, we measured their overlap using published ChromHMM segmentation from fibroblasts, downloaded through UCSC Genome Browser (Ernst et al. 2011). Hypermethylated and hypomethylated DMRs are both enriched for regulatory elements, including strong enhancers and active promoters (Fig. 3C). This enrichment increases in DMRs with larger differences in methylation, suggesting functional relevance (Supplemental Fig. S2G,H). To confirm the association between DMRs and active regulatory regions, we partitioned methylomes into CpG-poor low methylated regions (LMRs) and CpG-rich, fully unmethylated regions (UMRs), which correspond to proximal (promoter) and distal regulatory (enhancer) sites (Supplemental Fig. S3A-E; Stadler et al. 2011; Burger et al. 2013). Notably, 39\% of hypermethylated DMRs and $42 \%$ of hypomethylated DMRs were located within LMRs and UMRs that are specific to either NPFs or CAFs, respectively (Fig. $3 \mathrm{D})$. For example, the hypomethylated DMR in the gene body of CD9 occurs at a CAF-specific LMR and aligns with a region marked by the active histone modification H3K27ac (Fig. 3E). We also observed that an additional $\sim 30 \%$ of DMRs in CAFs extended from LMRs or UMRs preexisting in NPFs (Fig. 3D). For example, the hypomethylated DMR in IGFBP2 arises from a shared UMR that extends an additional $\sim 1800 \mathrm{bp}$ in CAFs (Fig. 3F). Collectively, these analyses show that methylation differences identified in CAFs are significantly enriched at unique regulatory regions.

\section{Differentially methylated regions in CAFs associate with functional changes}

Given that DMRs lie in regulatory regions, we next investigated whether they are associated with genes in biologically relevant pathways using the Genomic Regions Enrichment of Annotations Tool (GREAT) (Fig. 4A,B; McLean et al. 2010). Among DMRs with at least $30 \%$ difference in methylation levels, we observed enrichment of terms related to developmental processes and transcription factor binding, predominantly comprising genes from the T-box (TBX), forkhead box (FOX), and homeobox (HOX) gene families. We also observed enrichment of terms related to ligand-activated cell signaling, including the TGFbeta pathway and estrogen receptor $(E R \alpha)$ signaling.

To further assess the functional significance of the DMRs, we used stranded RNA-seq to determine whether the nearest proteincoding gene to each DMR was differentially expressed between CAFs and NPFs. We identified 445 DMRs associated with 220 differentially expressed genes $(P<0.05$, absolute fold change $\geq 1.5$ ) and termed these sites differentially expressed DMRs (DE-DMRs) (Supplemental Table S3). We confirmed gene expression differences in cross-platform validation with Affymetrix Human Gene 1.0ST Arrays (Supplemental Fig. S4A). The 162 DE-DMR genes that are more highly expressed in CAFs are predominantly associated with hypomethylated DMRs (278 hypomethylated vs. 74 hypermethylated), whereas the 58 DE-DMR genes with decreased expression in CAFs are more commonly associated with hypermethylated DMRs (36 hypomethylated vs. 57 hypermethylated) (Fig. $5 A)$. Compared with the full set of DMRs, DE-DMRs have significantly greater difference in methylation between CAFs and NPFs (permutation test, $P=1 \times 10^{-4}$ hypermethylated, and $P=1.05 \times$ $10^{-2}$ hypomethylated), but no difference in $\mathrm{CpG}$ density or genic distribution (Supplemental Table S4).

GREAT analysis of the DE-DMRs also revealed strong enrichment for developmental processes and genes associated with extracellular matrix (Supplemental Fig. S5A). A complementary analysis using Ingenuity Pathway Analysis (IPA) identified seven networks for the hypermethylated DE-DMRs and 14 networks for the hypomethylated DE-DMRs (Supplemental Table S5) with enrichment for diseases and biological functions, including cancer and organismal development (Supplemental Fig. S5B). Predicted upstream regulators of DE-DMR genes included members of the TGFbeta family (Supplemental Table S5).

\section{Functional DMRs define cell-type specificity between CAFs and NPFs across independent patients}

We evaluated the cell-type specificity of DE-DMRs in CAFs and NPFs from independent patients using two approaches. First, we

\section{Genome Research}

www.genome.org 
A

A $\quad \mathrm{CpG} \Gamma_{\mathrm{TSS}}$

CpG island $\square$ Promoter

Non CpG island
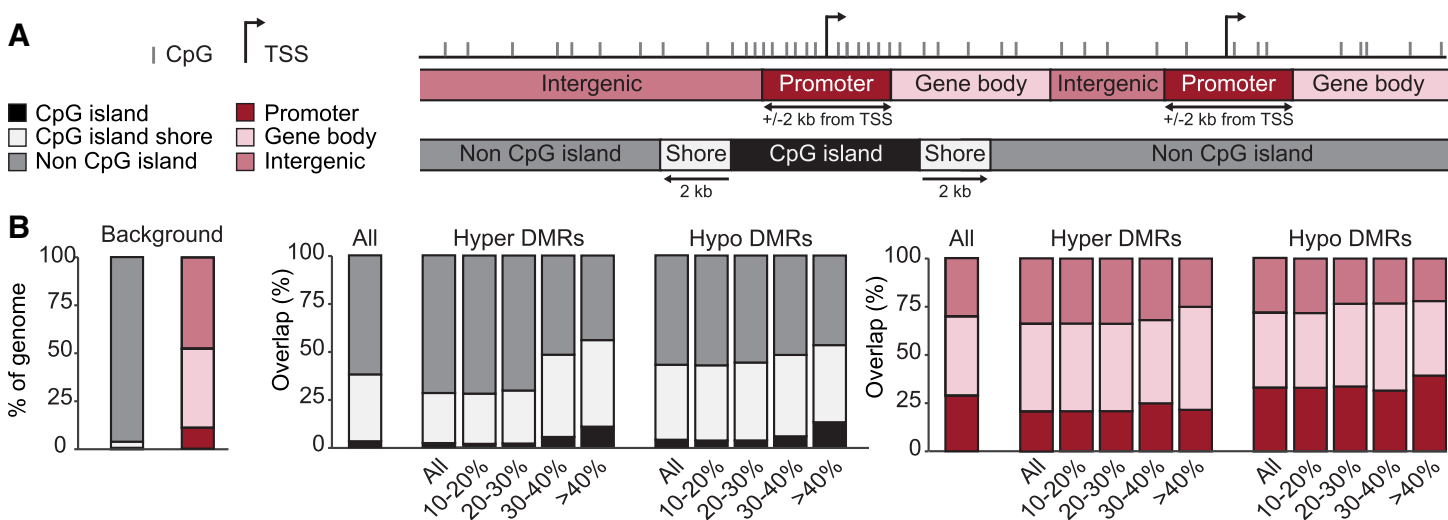

C

Observed/Expected

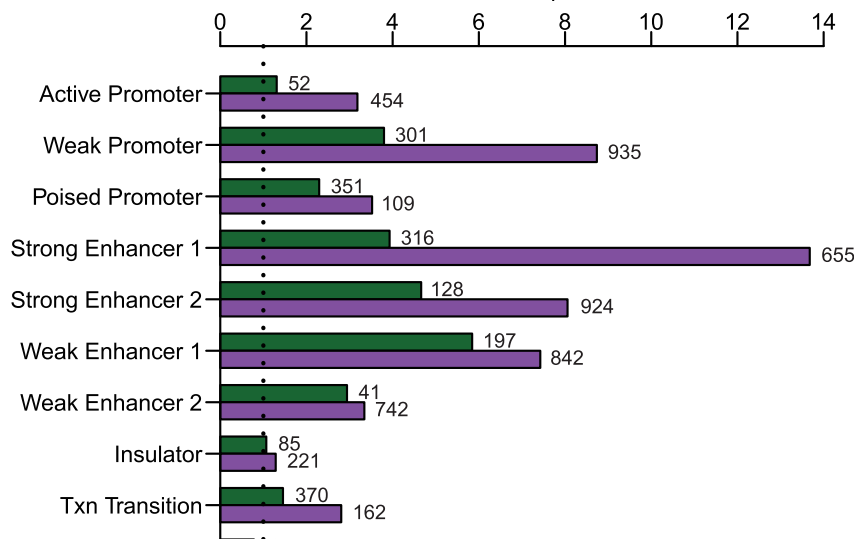

D

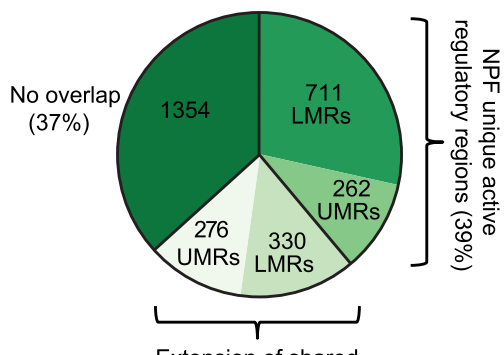

Extension of shared active regulatory regions $(24 \%)$

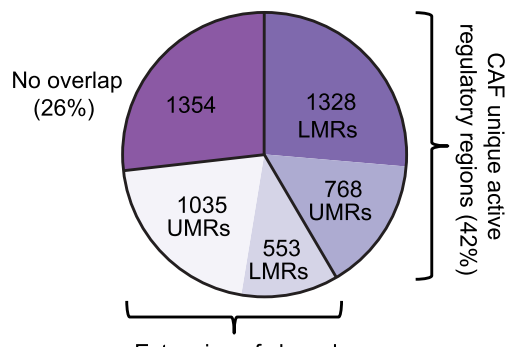

Hypermethylated DMR

Txn Elongation $\begin{array}{r}\cdot 168 \\ \cdot 236\end{array}$

Weak Txn $\begin{gathered}90 \\ \end{gathered}$

Repressed \begin{tabular}{c}
559 \\
\cline { 2 - 2 }
\end{tabular}

Heterochrom/lo $\square: 4058$

Repetitive/CNV 1 1739

Repetitive/CNV 2 :

E

Hypomethylated DMRs

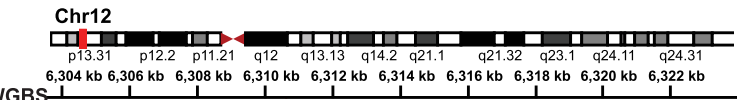

F

Extension of shared active regulatory regions $(32 \%)$

Chr2
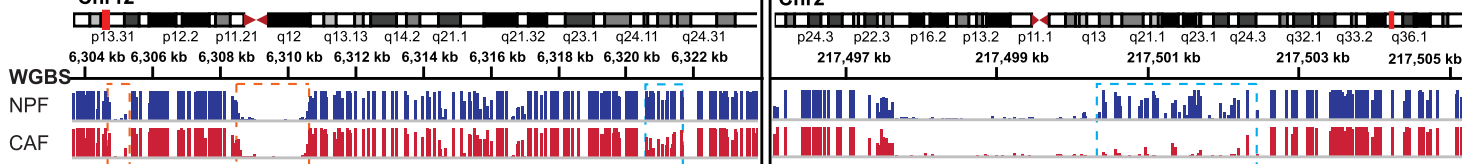

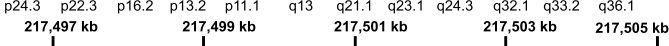

CAF-NPF

Hyper DMR

Hypo DMR

NPF UMR

CAF UMR

NPF LMR -

CAF LMR -

CpG islands

CD9

ENCODE H3K27ac

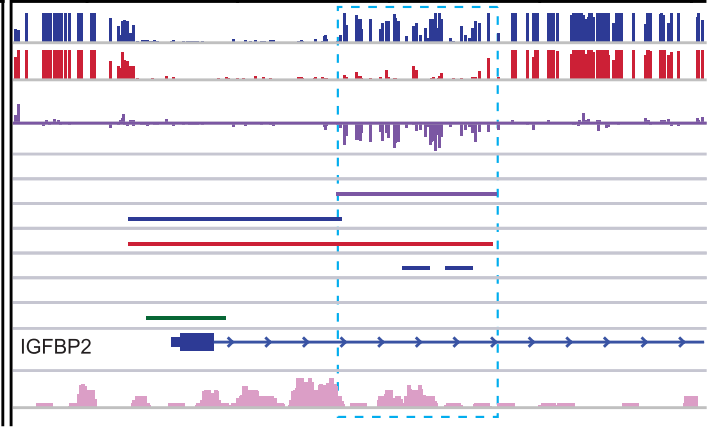

Figure 3. DMRs occur at regulatory regions. $(A)$ Schematic showing the genomic regions investigated in $B$. (B) The distribution of all CAF-NPF DMRs, and CAF-NPF DMRs grouped by percentage change in methylation, relative to each type of genomic region in $A$. (C) Comparison of CAF DMRs with ChromHMM data from normal fibroblasts. Numbers denote the number of DMRs associated with each type of region. Note that ENCODE defines two types of strong enhancers, weak enhancers, and repetitive/CNV regions. (D) The proportions of DMRs associated with LMRs and UMRs that are unique to NPFs (for hypermethylated DMRs) or CAFs (for hypomethylated DMRs) or that extend from LMRs and UMRs that are shared by NPFs and CAFs. (E) Pooled WGBS data ( $n=4$ patients) showing a hypomethylated DMR in the CD9 gene (blue box) occurring at a regulatory region defined by ChromHMM H3K27ac peaks (pink track) and a unique CAF LMR. Shared UMRs and LMRs between CAFs and NPFs are also shown (orange boxes). $(F)$ A hypomethylated DMR in IGFBP2 (blue box) arising from a shared UMR that extends in CAFs. The DMR lies in a regulatory region defined by ChromHMM H3K27ac peaks. 

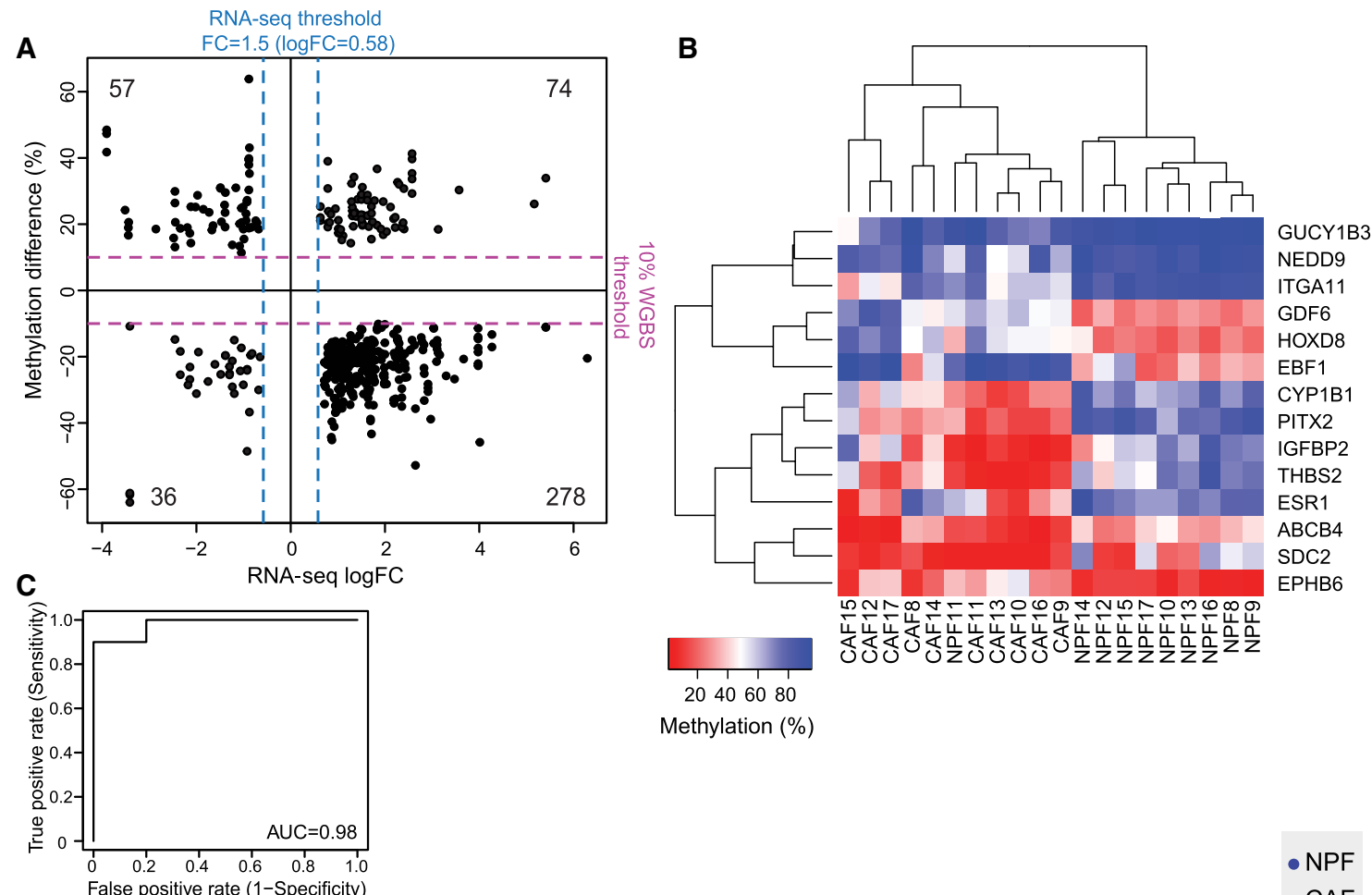

\section{EBF1}

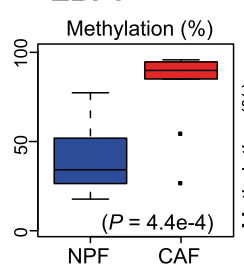

Chr 5 158.53262mb Methylation (\%)

\section{E EPHB6}
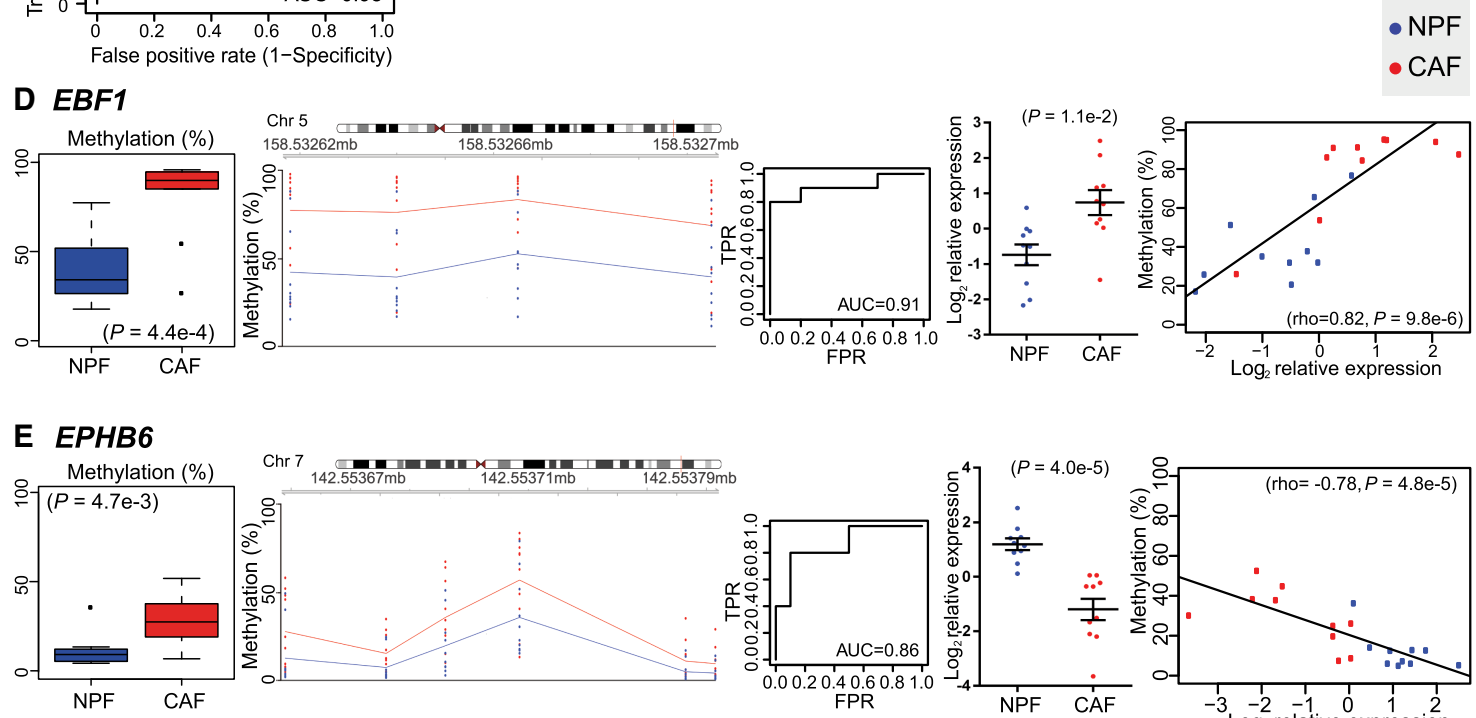

\section{F HOXD8}
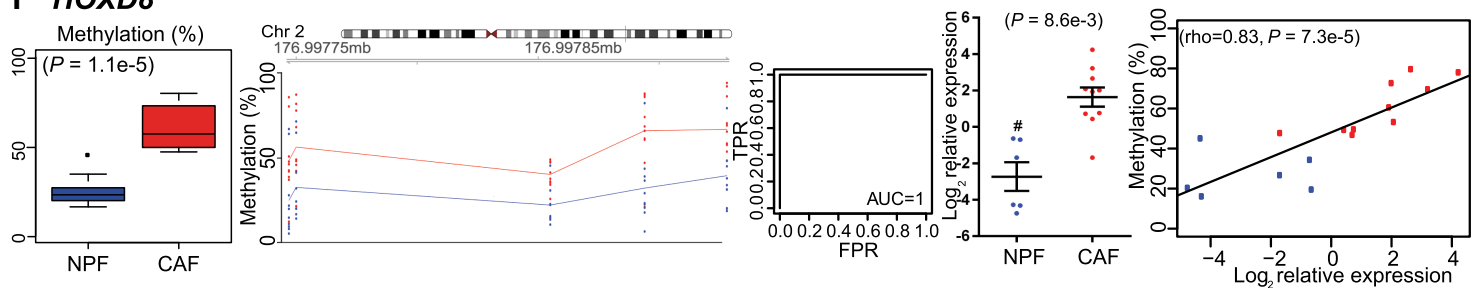

Figure 5. Consistent changes in DMRs associated with differentially expressed genes (DE-DMRs) between patients. ( $A$ ) Scatter plot of DE-DMRs showing the average percentage difference in DNA methylation versus average log fold change (logFC) in expression ( $n=4$ CAF-NPF pairs). Dotted lines indicate the thresholds for DE-DMRs. (B) Nonhierarchical clustering of CAFs and NPFs from 10 independent patients based on the methylation status of 14 candidate DEDMRs. The heatmap shows the percentage of DNA methylation of each DE-DMR in each sample. (C) Receiver operating characteristic (ROC) curve analysis based on the DNA methylation status of the 14 candidate DE-DMRs (AUC $=0.98, n=10$ CAF-NPF pairs). Representative examples of DE-DMRs in the EBF1 $(D), E P H B 6(E)$, and HOXD8 $(F)$ genes ([blue] NPF; [red] CAF), showing the average methylation across the amplicon in CAFs versus NPFs (one-sided paired $t$ test), the percentage of DNA methylation at each CpG site in each sample (trendlines denote the mean), ROC curves, the relative gene expression in each sample (with mean \pm SEM, one-sided $t$-test), and the significant correlation between methylation and expression (Spearman's test). The \# symbol denotes that HOXD8 expression was below the detection limit for NPF10, NPF13, NPF15, and NPF17; therefore, $n=6$ for NPFs and $n=10$ for CAFs. 
performed genome-wide comparisons with three additional patients using $450 \mathrm{~K}$ methylation BeadChips and gene expression arrays. Of the DE-DMRs covered by the $450 \mathrm{~K}$ arrays, $90 \%$ of probes $(120 / 145)$ validated the WGBS data (Supplemental Fig. S4B). Similarly, a high proportion $(72 \% ; 150 / 209)$ of the DE-DMR genes targeted by the expression array were consistent with the RNA-seq data (Supplemental Fig. S4C). Moreover we observed a strong correlation in the average CAF-NPF methylation and expression differences between the original and additional patients (DNA methylation $r=0.84, P<2.2 \times 10^{-16}$; gene expression: $r=0.53$, $P<2.2 \times 10^{-16}$ ) (Supplemental Fig. S4D,E).

Our second approach to validate DE-DMRs was to examine candidate genes using targeted bisulfite sequencing and quantitative RT-PCR for 10 new, extensively validated, patientmatched pairs of CAFs and NPFs. We selected 14 candidate genes with coordinated changes in methylation and expression and known roles in prostate cancer and/or the tumor microenvironment (summarized in Supplemental Table S6). All candidate regions showed a significant change in methylation $(P<0.05)$ in agreement with the WGBS data (Fig. 5B-F; Supplemental Fig. S6A,B; Supplemental Table S7). Notably, nonhierarchical clustering and ROC analysis based on these loci both correctly distinguished between CAFs and NPFs (19/20 fibroblast cultures, AUC 0.98) (Fig. 5B,C). Methylation levels at individual DMRs also discriminated between CAFs and NPFs, including HOXD8 with AUC=1 (Fig. 5D-F; Supplemental Fig. S6C; Supplemental Table S7). We also found that 13 of 14 candidate genes were significantly differentially expressed in CAFs versus NPFs $(P<0.05)$ (Fig. 5D-F; Supplemental Fig. S6D). There was a strong negative correlation between expression and methylation for IGFBP2, ITGA11, ESR1, and EPHB6, all of which have DMRs downstream from their promoter. In contrast, there were strong positive correlations for PITX2, with a DMR in the last intron, and HOXD8 and EBF1, which have intergenic DMRs, suggesting a mechanistic link between methylation and expression at these loci (Fig. 5D-F; Supplemental Fig. S6E). Collectively, our genome-wide and targeted validation, in independent patients, demonstrate that DE-DMRs are highly consistent between patients and define cell-type specificity between CAFs and NPFs.

\section{Diagnostic and prognostic value of tumor-specific DMRs}

We next asked whether the DMRs in CAFs are shared by epithelial prostate cancer cells. For this we used WGBS data from LNCaP prostate cancer epithelial cells and PrEC normal prostate epithelial cells (Fig. 6A) to identify CAF-NPF DMRs that showed a mean DNA methylation difference $>30 \%$ in LNCaP compared to PrEC. This revealed that $10 \%$ of CAF-NPF DMRs showed robust DNA methylation alterations in LNCaP versus PrEC cells $(n=493$ / 5038 hypomethylated, $n=294 / 2496$, hypermethylated) (Fig. 6B; Supplemental Table S8). We defined these regions as tumor-specific DMRs (tsDMRs). GREAT analysis showed that the tsDMRs were significantly enriched in biological processes associated with development (with genes including T-box and HOX genes) and endocrine hormone secretion (Supplemental Fig. S7A,B). Furthermore, Ingenuity Pathway Analysis showed that the tsDMRs were overrepresented in genes associated with cancer and reproductive system disease, and analysis of the upstream regulators of these genes predicted regulation by the estrogen receptor and members of the TGFbeta pathway (Supplemental Fig. S7C; Supplemental Table S5).
Second, to explore the potential clinical relevance of tsDMRs, we investigated methylation profiles of clinical prostate cancer specimens, which contain a heterogeneous mix of epithelium and stroma, a confounding feature for many diagnostic signatures (Isella et al. 2015). We used publicly available $450 \mathrm{~K}$ methylation data of tumor and normal prostate specimens from The Cancer Genome Atlas (TCGA) (The Cancer Genome Atlas Research Network 2015). Of the subset of tsDMRs covered by $450 \mathrm{~K}$ probes, $83 \%(91 / 109)$ of hypermethylated tsDMRs and 65\% (60/92) of hypomethylated tsDMRs showed consistent methylation differences (FDR < 0.05) in the TCGA samples (Fig. 6A,B; Supplemental Table S9). Furthermore, ROC analysis showed that 50 hypermethylated tsDMRs and 15 hypomethylated tsDMRs accurately discriminated between the tumor and normal samples with AUC $>0.8$ (Supplemental Table S9; Supplemental Fig. S8).

Some of the most striking tsDMRs flank the TBX3 gene encoding a developmental transcriptional repressor. We found concordant hypermethylated DMRs in CAFs and LNCaP cells that truncate a UMR covering TBX3 (Fig. 7A). Importantly, WBGS data from four clinical samples confirmed that the tsDMRs are consistently hypermethylated in tumors compared to matched nonmalignant prostate tissue, as did $450 \mathrm{~K}$ data from TCGA (Fig. 7B). Collectively, the TBX3 tsDMRs discriminated between normal and cancer samples in TCGA with an AUC of 0.84 (Fig. 7C); however, individual tsDMRs had AUCs of up to 0.92 (Supplemental Table S9). Other notable tsDMRs exist in regulatory enhancer regions of the ETS Homologous Factor (EHF), Mutated in Colorectal Cancer (MCC), and STEAP2 (Prostate Cancer Associated Protein 1) genes (Supplemental Fig. S8). These tsDMRs all had consistent changes in DNA methylation across WGBS data from CAFs, LNCaP cells, and tumor tissue, and $450 \mathrm{~K}$ data from TCGA. Unlike previous studies of prostate CAFs and tumor stroma (Hanson et al. 2006; Rodriguez-Canales et al. 2007; Banerjee et al. 2014), the GSTP1 CpG island promoter was only hypermethylated in prostate cancer epithelial cells and not in CAFs (Fig. 7D).

The abundance of reactive stroma in prostate cancer has previously been linked to recurrence-free survival (Ayala et al. 2003), so we explored the possible association of tsDMRs with patient prognosis. We used two publicly available gene expression data sets (Glinsky et al. 2004; Taylor et al. 2010), as comparable cohorts with methylation data and extensive clinical follow-up are not currently available. We identified tsDMRs associated with gene expression changes and examined their relationship with recurrence-free survival (Supplemental Methods). We identified an intronic hypermethylated tsDMR in the CCDC68 gene, which was also consistently down-regulated in CAFs, LNCaPs, and TCGA tumor samples (Fig. 8A-C). CCDC68 was recently identified as a tumor suppressor gene in pancreatic cancer and functional studies showed that decreased CCDC68 expression increases tumor growth (Radulovich et al. 2015). Since DNA methylation of TSS probes surrounding the tsDMR and transcript abundance of CCDC68 are highly correlated (Fig. 8D), we used CCDC68 expression as a surrogate for the tsDMR in Kaplan-Meier analysis. In agreement with the decreased expression and hypermethylation of CCDC68 in CAFs and LNCaP cells, patients with lower CCDC68 gene expression showed significantly decreased recurrence-free survival compared to patients with high CCDC68 expression (log-rank test, $P=0.012$, hazard ratio $(\mathrm{HR})=2.798,95 \%$ confidence interval $(\mathrm{CI})=1.252-6.251$ ) (Fig. 8E), suggesting that some tsDMRs may also be associated with patient prognosis. We further confirmed this association using gene expression data

\section{Genome Research}

www.genome.org 


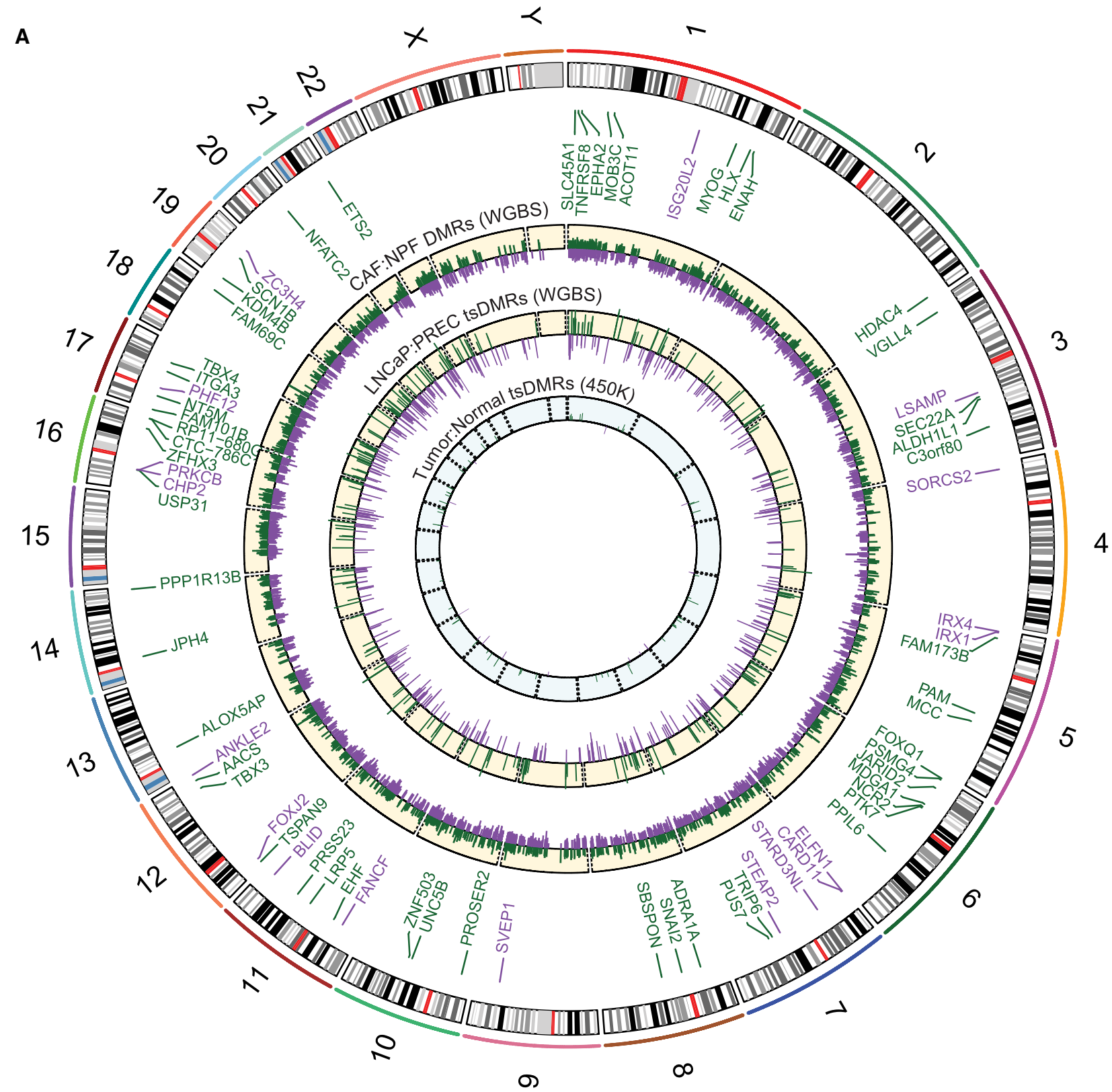

B
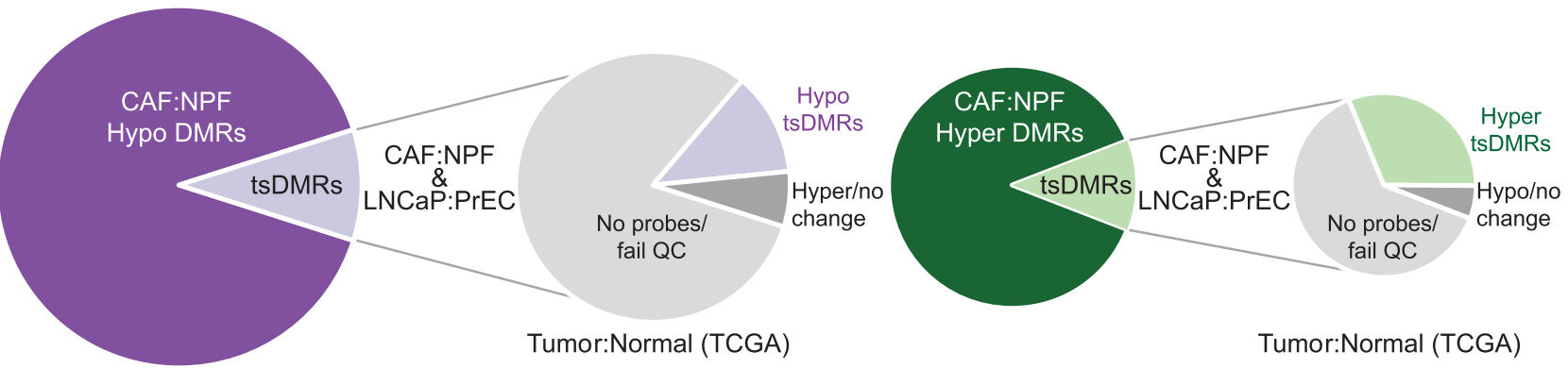

Figure 6. Tumor-specific DMRs common to CAFs and cancer cells. (A) Circos plot showing common DMRs identified by WGBS of CAF versus NPF, WGBS of LNCaP versus PrEC cells (middle), and 450K arrays of cancer versus normal tissue from TCGA (inner). (Green) Hypermethylated; (purple) hypomethylated. The height of each track represents a 50\% methylation difference. Note that only tsDMRs are shown for LNCaP:PrEC and TCGA samples. (B) Pie charts showing the proportion of DMRs that are tsDMRs and that are consistent with 450K data from TCGA. 
Pidsley et al.

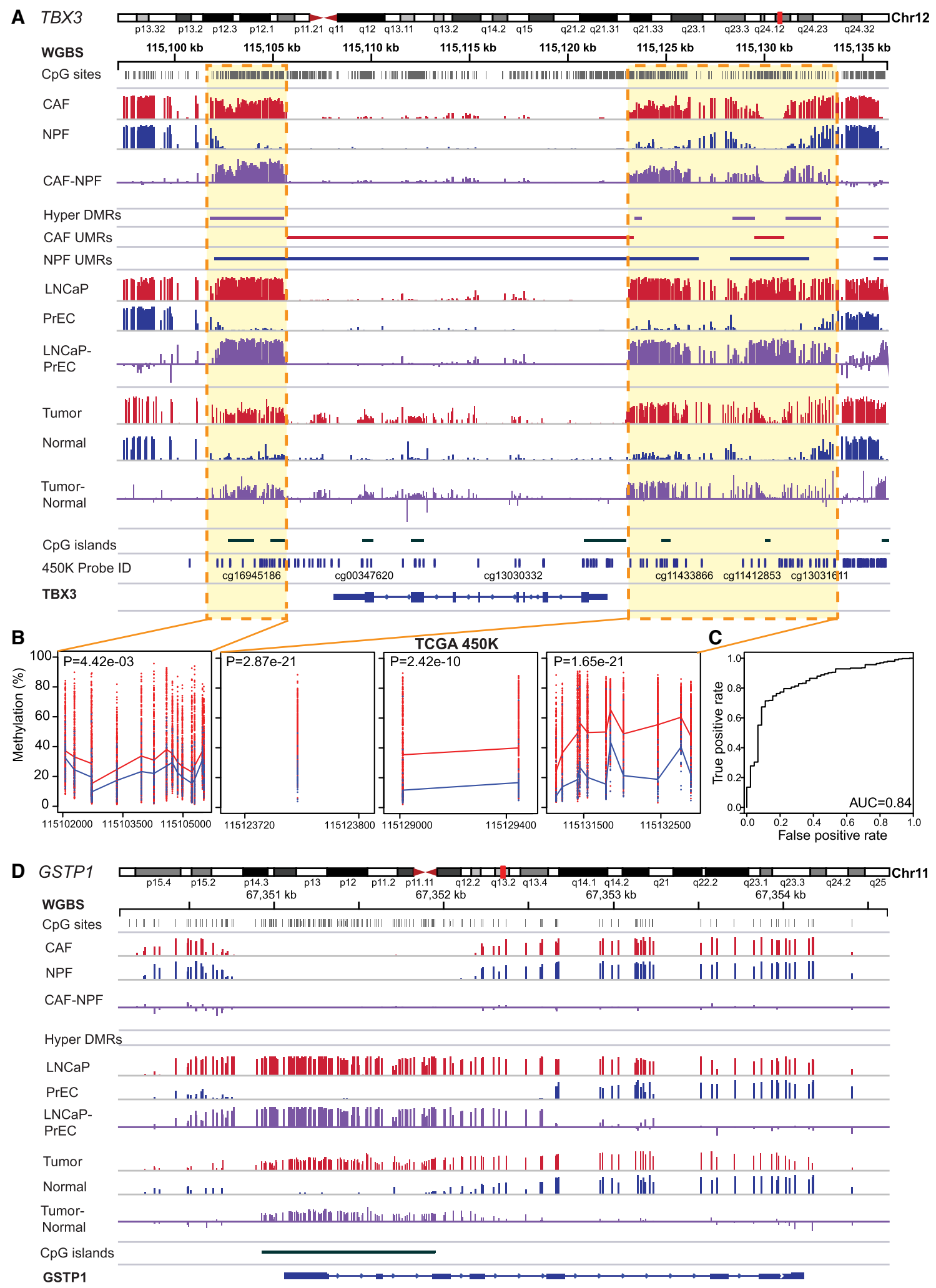

Figure 7. Diagnostic value of tumor-specific DMRs. ( $A$ ) WGBS data for TBX3 in fibroblasts (CAFs vs. NPFs, $n=4$ pairs), epithelial cells (LNCaP vs. PrEC), and patient tissue (matched tumor vs. normal) showing consistent hypermethylated tsDMRs in each data set. (B) 450K data from TCGA is also shown with dots indicating the DNA methylation status of tumor (red, $n=392$ ) and normal (blue, $n=45$ ) samples at each probe (trendlines denote mean methylation). (C) An ROC curve showing that the average methylation status of the four TBX3 tsDMRs discriminates between normal and cancer tissues in TCGA. (D) WGBS data showing lack of hypermethylation of the GSTP1 promoter CpG island in CAFs versus NPFs, unlike LNCaP versus PrEC and matched tumor versus normal patient tissues.

\section{Genome Research}

www.genome.org 
A
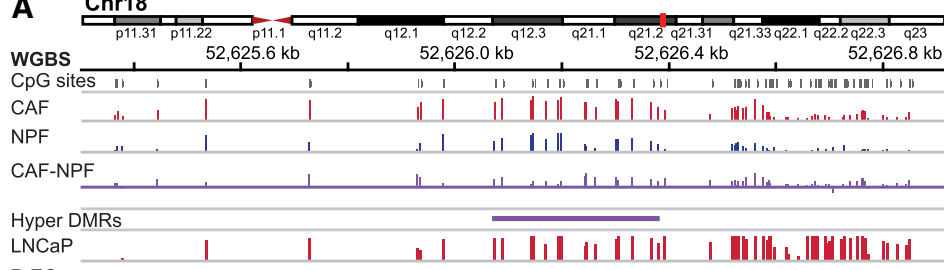

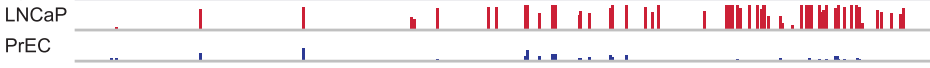

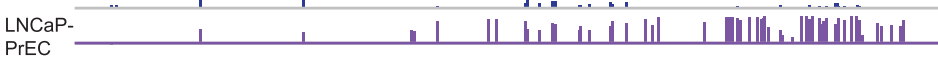

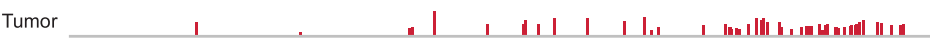

$\begin{array}{llllll}\text { Normal } & 1 & 1 & 1 & \ldots & \ldots \ldots \ldots\end{array}$

Tumor-
Normal

$\mathrm{CpG}$ islands

cg07058998 cg16583884

$450 \mathrm{~K}$

Probe ID ' $\operatorname{cg} 12113740$

cg07177852, cg12974637 cg07058998

cg12955441 cg03778029
B

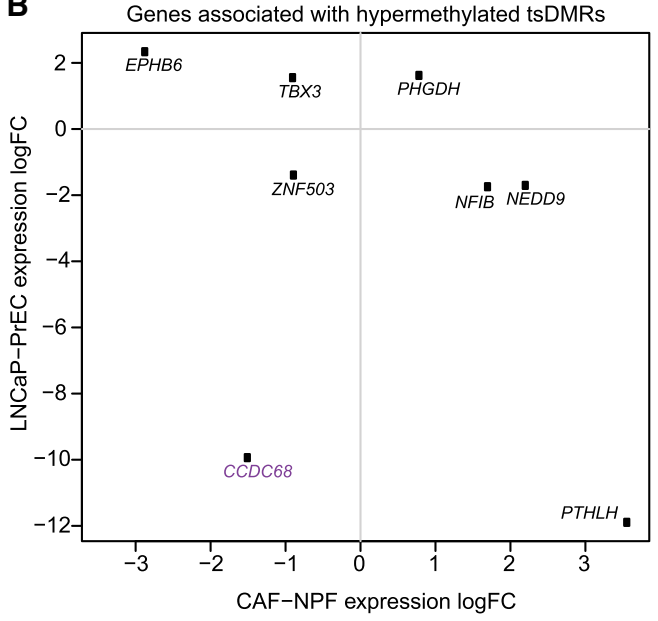

C

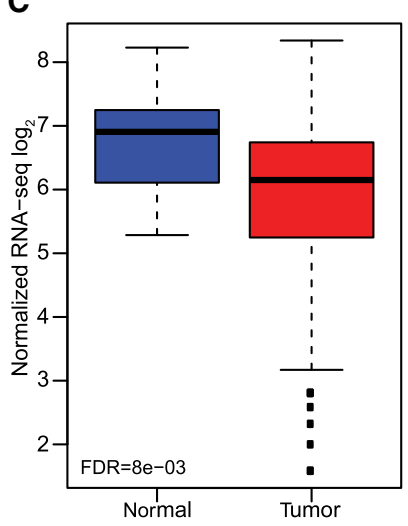

D

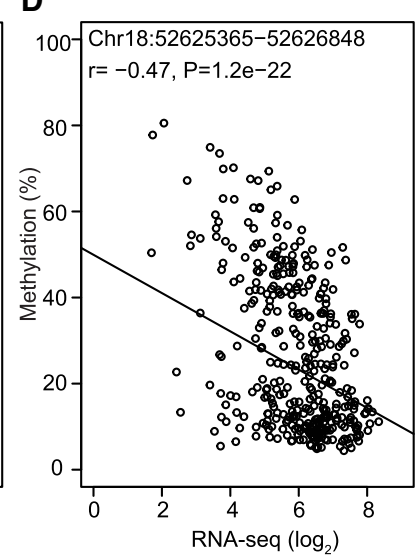

$\mathbf{E}_{1}$

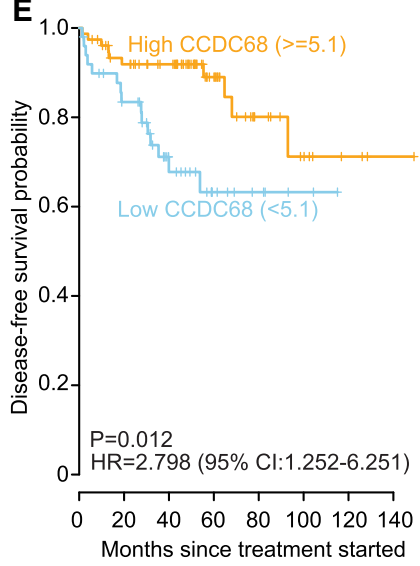

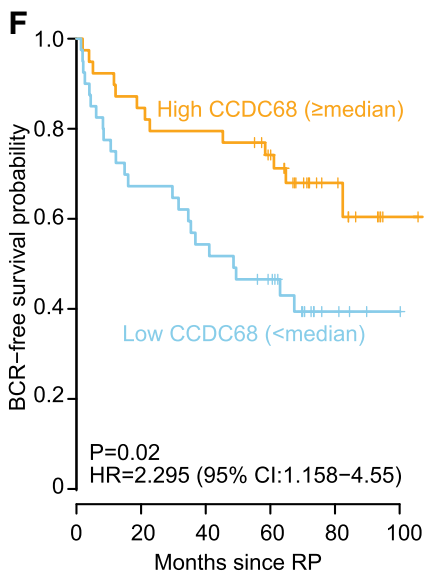

Figure 8. $C C D C 68$ has prognostic value in localized prostate cancer. $(A)$ WGBS data showing consistent hypermethylation of CCDC68 in CAFs versus NPFs ( $n=4$ pairs), LNCaP versus PrEC, and matched tumor versus normal tissues ( $n=4$ pairs). The locations of $450 \mathrm{~K}$ probes are shown in gray. (B) Scatter plot of differentially expressed genes associated with hypermethylated tsDMRs. CCDC68 (purple) is down-regulated in CAFs versus NPFs ( $n=4$ pairs) and also in LNCaP versus PrEC cells. (C) Box plot showing decreased CCDC68 expression in tumor $(n=392)$ versus normal $(n=45)$ tissue samples from TCGA. (D) The relationship between 450K methylation averaged across all probes at the TSS of CCDC68 and gene expression for TCGA tumor specimens. $(E, F)$ Kaplan-Meier curves showing low (blue) CCDC68 expression is associated with poor recurrence-free survival compared to high CCDC68 expression (orange) in Taylor $(E)$ and Glinsky $(F)$ data sets $(n=127$ and $n=79$ respectively, log-rank test).

from an additional cohort (log-rank test, $P=0.02, \mathrm{HR}=2.295,95 \%$ $\mathrm{CI}=1.158-4.55$ ) (Fig. 8F; Glinsky et al. 2004).

\section{Discussion}

Genomic and epigenetic aberrations in tumor epithelia are well described, but the molecular changes in the surrounding microenvironment, predominated by CAFs, are controversial (Campbell et al. 2009). Here, we generated high-resolution methylome maps of purified CAFs and matched NPFs. We found no evidence of gross genomic alterations in CAFs, similar to other reports (Qiu et al. 2008; Bianchi-Frias et al. 2016). Instead, CAFs harbor discrete and consistent DNA methylation differences, predominantly at regulatory regions across the genome. We identified a core set of genes with coordinated changes in DNA methylation and mRNA expression (DE-DMRs), including genes involved in tissue development and stromal-epithelial cell signaling. These DE-DMRs distinguished between CAFs and NPFs with high sensitivity and specificity across patients. Further, we identified common DMRs in CAFs and prostate cancer cells with diagnostic potential. Collectively our data demonstrate that the phenotype of CAFs is not merely a transient reaction to adjacent cancer epithelium, but involves enduring and recurrent epigenome changes.

Previous studies reported widespread hypomethylation in CAFs, but with techniques that do not assess the entire genome (Jiang et al. 2008; Vizoso et al. 2015). Using WGBS, we revealed that CAFs have locus-specific changes in DNA methylation rather than the global loss of DNA methylation that commonly occurs in cancer epithelial cells. We detected more hypomethylated than hypermethylated DMRs, but do not classify this as global hypomethylation since they span a small fraction of the genome. Notably, we found that many DMRs are at regulatory regions, including promoters and enhancers, which are not interrogated by lower resolution methods. The presence of DMRs in promoters is consistent with reported differences in transcription factor binding at transcriptional start sites between prostate CAFs and NPFs (Leach 
et al. 2017; Nash et al. 2017). Many of the CAF-associated DMRs also create ectopic enhancer (LMRs) and promoter (UMRs) elements, and more than a third extend preexisting LMRs or UMRs, indicating a cancer-associated spread of hyper- or hypomethylation at the border of these regulatory elements. However, further analysis is required to address if there are resulting alterations in enhancer-promoter chromatin interactions.

DMRs are extremely consistent in CAFs, with high concordance across different experimental platforms and independent patient samples. This is remarkable given that the CAFs were derived from patients with a range of clinical features and the considerable inter-patient tumor and stoma heterogeneity reported in localized sporadic prostate cancer (Franco et al. 2011; Kiskowski et al. 2011; The Cancer Genome Atlas Research Network 2015). One of the challenges in the field is to validate CAFs without using laborious functional assays. However, we found that a core set of DMRs could distinguish between CAFs and NPFs with high sensitivity and specificity, providing the first epigenetic signature for these two cell types. This signature may also provide a way to estimate the proportion of CAFs in patient tissue in future studies.

Strikingly, a subset of DMRs in CAFs is also shared with DMRs identified in prostate cancer epithelial cells. These newly defined tumor-specific (ts) DMRs were consistent between different patients and accurately distinguished normal from tumor tissue. The tsDMRs transcend the considerable global differences in DNA methylation between each cell type, since, unlike CAFs, cancer cells undergo extensive hypomethylation (Hansen et al. 2011; Berman et al. 2012; Kretzmer et al. 2015). Notably, the tsDMRs are strongly overrepresented in developmental gene families and genes involved in endocrine hormone secretion, such as TBX3 (Holterhus et al. 2007; Beukers et al. 2015), suggesting that they are associated with functional roles in cancer progression. Indeed specific tsDMRs were found in known tumor suppressor genes (for example, EHF [Albino et al. 2012] and MCC [KohonenCorish et al. 2007]) and oncogenes (for example, STEAP2 [Whiteland et al. 2014]). Collectively, the tsDMRs suggest a convergent malignant epigenetic pathway across cell types. We propose that tsDMRs may be used as tumor microenvironmentbased biomarkers for detecting prostate cancer in heterogeneous clinical samples and biopsies that contain a mix of epithelium and stroma. In summary, our study has wide ramifications for cancer diagnosis, as it highlights that it is not only the cancer epigenome that is remodeled, but that CAFs are also epigenetically altered, giving rise to a new epigenetic hallmark of the cancer microenvironment that promises to have clinical utility.

\section{Methods}

\section{Patient tissue}

Primary fibroblasts ( $n=17)$ were isolated from radical prostatectomy (RP) specimens collected with human ethics approval from Monash University (2004/145), Cabrini Hospital (03-14-04-08), and Epworth Hospital (53611). For WGBS, biopsies were taken from four patients' RP tissue, retrieved with human ethics approval from the Garvan Institute/St Vincent's Prostate Cancer biobank (SVH File Number 12/231). See Supplemental Methods for more details.

\section{Cell culture and validation of prostatic fibroblasts}

Primary cultures of NPFs and CAFs from matched nonmalignant and cancer regions of RP tissue, respectively, were established and validated as previously described (Clark et al. 2013; Lawrence et al. 2013; Niranjan et al. 2013). See Supplemental Methods for more details.

\section{Whole-genome bisulfite sequencing}

Library preparation of matched CAF-NPF samples ( $n=4$ pairs) and LNCaP and PrEC cell lines was performed using the Illumina Paired-end DNA Sample Prep Kit (Illumina) and sequencing performed on the Illumina HiSeq 2500 platform. FFPE tumor and matched adjacent normal tissue ( $n=4$ pairs) WGBS libraries were prepared using the EpiGnome Methyl-Seq kit (Epicentre). See Supplemental Methods for more details about library preparation and data processing.

\section{WGBS statistical analysis}

The R package bsseq (Hansen et al. 2012) was used to identify CAF-NPF DMRs. UMRs and LMRs were identified using the MethylSeekR Bioconductor package (Burger et al. 2013). See Supplemental Methods for more details.

\section{Enrichment analysis for chromatin states}

A BED-formatted annotation file of chromatin states for a normal fibroblast cell line was downloaded through UCSC Genome Browser (Ernst et al. 2011). The Genomic Association Tester (GAT) simulation framework was used to assess enrichment of WGBS DMRs in each chromatin state (Heger et al. 2013). See Supplemental Methods for more details.

\section{Microarray genome-wide DNA methylation analysis}

DNA from seven patient-matched CAF-NPF pairs was bisulfite treated using the EZ-96 DNA methylation kit (Zymo Research). DNA methylation was quantified using the Infinium HumanMethylation450 (450K) BeadChip (Illumina) run on the HiScan System (Illumina). QC and processing were performed using the minfi package in R v1.12.0 (Aryee et al. 2014). See Supplemental Methods for more details.

\section{TCGA microarray genome-wide DNA methylation}

Raw IDAT files and corresponding clinical and specimen data for prostate adenocarcinoma samples were downloaded from https:// tcga-data.nci.nih.gov/tcgafiles on May 26, 2015 (TCGA data have now been moved to https://portal.gdc.cancer.gov). We calculated the mean methylation level for each tsDMR and then ascertained the methylation difference between matched-tumor normal pairs using paired $t$-tests. The receiver operating characteristic (ROC) curve and area under the curve (AUC) were calculated using ROCR v1.0-7 (Sing et al. 2005). See Supplemental Methods for more details.

\section{Bisulfite-amplicon sequencing with MiSeq}

Independent verification analysis was performed on 10 additional patient-matched CAF-NPF pairs. Bisulfite-PCR amplification was performed using primers designed with Sequenom EpiDesigner software (https://www.epidesigner.com) and conditions described in Supplemental Table S10. Libraries were prepared using the TruSeq DNA PCR-Free Low Sample Preparation Kit (Illumina). See Supplemental Methods for more details about library preparation and data processing.

\section{Genome Research}

www.genome.org 


\section{RNA sequencing}

CAF-NPF RNA ( $n=4$ pairs) was depleted of ribosomal RNA using RiboZero (Illumina). Libraries were prepared using the TruSeq Stranded Total RNA sample kit (Illumina), followed by directional 75-bp paired-end sequencing. See Supplemental Methods for more details. We used in-house LNCaP and PrEC RNA-seq data (Taberlay et al. 2016). All raw and processed data are publicly available at https://www.ncbi.nlm.nih.gov/geo under accession number GSE73784.

\section{Microarray-based genome-wide expression analysis}

CAF-NPF RNA ( $n=7$ pairs) was analyzed using the Human Gene 1.0 ST array (Affymetrix) in two sets. See Supplemental Methods for more details.

\section{Quantitative RT-PCR}

Total RNA was reverse-transcribed using SuperScript III (Invitrogen). All primers are listed in Supplemental Table S11. See Supplemental Methods for more details.

\section{Microarray-based genome-wide genotyping analysis}

CAF-NPF DNA ( $n=4$ pairs) was used to assess genomic aberrations using Infinium Omni2.5-8 BeadChips (Illumina). See Supplemental Methods for more details.

\section{Gene ontology and pathway analysis}

GREAT was used to analyze the functional significance of DMRs (McLean et al. 2010). IPA software (IPA, QIAGEN) was used to map lists of identified genes to their corresponding gene object in the Ingenuity Knowledge Base. See Supplemental Methods for more details.

\section{Prognostic analysis}

Prognostic analysis was undertaken using TCGA PRAD processed RNA-seq V2 data, downloaded from https://tcga-data.nci.nih. gov/tcgafiles on April 19, 2016 (TCGA data have now been moved to https://portal.gdc.cancer.gov); RNA-seq data from the NCBI GEO database with accession GSE21032 (Taylor et al. 2010); and Affymetrix Human Genome U95Av2 array data from the ONCOMINE Database (Glinsky et al. 2004). See Supplemental Methods for more details.

\section{Data access}

The data generated as part of this study have been submitted to the NCBI Gene Expression Omnibus (GEO; https://www.ncbi. nlm.nih.gov/geo) under accession number GSE86260.

\section{Acknowledgments}

We thank the Epigenetics Research and Prostate Cancer Groups (Garvan Institute of Medical Research) and Prostate Cancer Research Group (Monash University and Peter MacCallum Cancer Centre) for helpful discussions; Dr. Brigid O'Gorman for help with figure preparation; Dr. Jeremy Grummet, Dr. Shomik Sengupta, and Dr. Ross Snow for patient recruitment; TissuPath Pathology for pathology support; the Australian Prostate Cancer BioResource for specimen collection; and the patients who donated their tissue. The results are based in part on data generated by the TCGA Research Network (http://cancergenome.nih.gov). This work was funded by Cancer Australia (1044458), the National
Health and Medical Research Council (Program 535903; Project grants 1070418 and 1106870; and Fellowships to S.J.C. [1063559], G.P.R. [1002648 and 1102752], M.G.L. [1035721], and R.J.D. [1058540]), Cancer Institute of New South Wales Fellowship to R.P. (14/ECF/1-23), Australian Prostate Cancer Research Centre-NSW, Prostate Cancer Foundation of Australia (Movember Young Investigator Grant to M.G.L. [YI0911]), Victorian Cancer Agency (Fellowships to R.A.T. and H.T.N.), RT Hall Trust, and TissuPath Pathology.

Author contributions: S.J.C., M.G.L., R.P., G.P.R., C.S., and R.A.T. conceived and designed the study. M.F., L.G.H., M.P., and P.S. were responsible for the identification and consent of patients. Pathology analysis was carried out by J.K., S.N., J.P., and A.R. P.B., R.M.C., M.G.L., R.P., T.P., B.N., S.N., W.Q., M.R., J.S., and H.W. performed the experiments. R.P., M.G.L., E.Z., A.S., N.J.A., H.F., and H.T.N. analyzed and interpreted the data. S.J.C., R.J.D., M.G.L., R.P., G.P.R., C.S., and R.A.T. supervised the research. S.J.C., M.G.L., R.P., and G.P.R wrote, reviewed, and revised the manuscript. All authors read and approved the final manuscript.

\section{References}

Albino D, Longoni N, Curti L, Mello-Grand M, Pinton S, Civenni G, Thalmann G, D'Ambrosio G, Sarti M, Sessa F, et al. 2012. ESE3/EHF controls epithelial cell differentiation and its loss leads to prostate tumors with mesenchymal and stem-like features. Cancer Res 72: 2889-2900.

Ao M, Franco OE, Park D, Raman D, Williams K, Hayward SW. 2007. Crosstalk between paracrine-acting cytokine and chemokine pathways promotes malignancy in benign human prostatic epithelium. Cancer Res 67: 4244-4253.

Aryee MJ, Jaffe AE, Corrada-Bravo H, Ladd-Acosta C, Feinberg AP, Hansen KD, Irizarry RA. 2014. Minfi: a flexible and comprehensive Bioconductor package for the analysis of Infinium DNA methylation microarrays. Bioinformatics 30: 1363-1369.

Ashida S, Orloff MS, Bebek G, Zhang L, Zheng P, Peehl DM, Eng C. 2012. Integrated analysis reveals critical genomic regions in prostate tumor microenvironment associated with clinicopathologic phenotypes. Clin Cancer Res 18: 1578-1587.

Ayala G, Tuxhorn JA, Wheeler TM, Frolov A, Scardino PT, Ohori M, Wheeler M, Spitler J, Rowley DR. 2003. Reactive stroma as a predictor of biochemical-free recurrence in prostate cancer. Clin Cancer Res 9: 4792-4801.

Banerjee J, Mishra R, Li X, Jackson RS II, Sharma A, Bhowmick NA. 2014. A reciprocal role of prostate cancer on stromal DNA damage. Oncogene 33: 4924-4931.

Berman BP, Weisenberger DJ, Aman JF, Hinoue T, Ramjan Z, Liu Y, Noushmehr H, Lange CP, van Dijk CM, Tollenaar RA, et al. 2012. Regions of focal DNA hypermethylation and long-range hypomethylation in colorectal cancer coincide with nuclear lamina-associated domains. Nat Genet 44: 40-46.

Beukers W, Kandimalla R, Masius RG, Vermeij M, Kranse R, van Leenders GJ, Zwarthoff EC. 2015. Stratification based on methylation of TBX2 and TBX3 into three molecular grades predicts progression in patients with pTa-bladder cancer. Mod Pathol 28: 515-522.

Bhowmick NA, Chytil A, Plieth D, Gorska AE, Dumont N, Shappell S, Washington MK, Neilson EG, Moses HL. 2004. TGF- $\beta$ signaling in fibroblasts modulates the oncogenic potential of adjacent epithelia. Science 303: $848-851$.

Bianchi-Frias D, Basom R, Delrow JJ, Coleman IM, Dakhova O, Qu X, Fang M, Franco OE, Ericson NG, Bielas JH, et al. 2016. Cells comprising the prostate cancer microenvironment lack recurrent clonal somatic genomic aberrations. Mol Cancer Res 14: 374-384.

Burger L, Gaidatzis D, Schübeler D, Stadler MB. 2013. Identification of active regulatory regions from DNA methylation data. Nucleic Acids Res 41: e155.

Campbell I, Polyak K, Haviv I. 2009. Clonal mutations in the cancer-associated fibroblasts: the case against genetic coevolution. Cancer Res 69: 6765-6769.

The Cancer Genome Atlas Research Network. 2015. The molecular taxonomy of primary prostate cancer. Cell 163: 1011-1025.

Clark AK, Taubenberger AV, Taylor RA, Niranjan B, Chea ZY, Zotenko E, Sieh S, Pedersen JS, Norden S, Frydenberg M, et al. 2013. A bioengineered microenvironment to quantitatively measure the tumorigenic properties of cancer-associated fibroblasts in human prostate cancer. Biomaterials 34: $4777-4785$. 
Dawsey SP, Roth MJ, Adams L, Hu N, Wang QH, Taylor PR, Woodson K. 2008. COX-2 (PTGS2) gene methylation in epithelial, subepithelial lymphocyte and stromal tissue compartments in a spectrum of esophageal squamous neoplasia. Cancer Detect Prev 32: 135-139.

Ernst J, Kheradpour P, Mikkelsen TS, Shoresh N, Ward LD, Epstein CB, Zhang X, Wang L, Issner R, Coyne M, et al. 2011. Mapping and analysis of chromatin state dynamics in nine human cell types. Nature 473: 43-49.

Fiegl H, Millinger S, Goebel G, Müller-Holzner E, Marth C, Laird PW, Widschwendter M. 2006. Breast cancer DNA methylation profiles in cancer cells and tumor stroma: association with HER-2/neu status in primary breast cancer. Cancer Res 66: 29-33.

Franco OE, Jiang M, Strand DW, Peacock J, Fernandez S, Jackson RS II, Revelo MP, Bhowmick NA, Hayward SW. 2011. Altered TGF- $\beta$ signaling in a subpopulation of human stromal cells promotes prostatic carcinogenesis. Cancer Res 71: 1272-1281.

Glinsky GV, Glinskii AB, Stephenson AJ, Hoffman RM, Gerald WL. 2004 Gene expression profiling predicts clinical outcome of prostate cancer. J Clin Invest 113: 913-923.

Hanahan D, Weinberg RA. 2011. Hallmarks of cancer: the next generation. Cell 144: 646-674.

Hansen KD, Timp W, Bravo HC, Sabunciyan S, Langmead B, McDonald OG, Wen B, Wu H, Liu Y, Diep D, et al. 2011. Increased methylation variation in epigenetic domains across cancer types. Nat Genet 43: 768-775.

Hansen KD, Langmead B, Irizarry RA. 2012. BSmooth: from whole genome bisulfite sequencing reads to differentially methylated regions. Genome Biol 13: R83.

Hanson JA, Gillespie JW, Grover A, Tangrea MA, Chuaqui RF, Emmert-Buck MR, Tangrea JA, Libutti SK, Linehan WM, Woodson KG. 2006. Gene promoter methylation in prostate tumor-associated stromal cells. J Natl Cancer Inst 98: 255-261.

Heger A, Webber C, Goodson M, Ponting CP, Lunter G. 2013. GAT: a simulation framework for testing the association of genomic intervals. Bioinformatics 29: 2046-2048.

Holterhus PM, Deppe U, Werner R, Richter-Unruh A, Bebermeier JH, Wünsch L, Krege S, Schweikert HU, Demeter J, Riepe F, et al. 2007. Intrinsic androgen-dependent gene expression patterns revealed by comparison of genital fibroblasts from normal males and individuals with complete and partial androgen insensitivity syndrome. BMC Genomics 8: 376.

Hu M, Yao J, Cai L, Bachman KE, van den Brûle F, Velculescu V, Polyak K. 2005. Distinct epigenetic changes in the stromal cells of breast cancers. Nat Genet 37: 899-905.

Isella C, Terrasi A, Bellomo SE, Petti C, Galatola G, Muratore A, Mellano A, Senetta R, Cassenti A, Sonetto C, et al. 2015. Stromal contribution to the colorectal cancer transcriptome. Nat Genet 47: 312-319.

Jiang L, Gonda TA, Gamble MV, Salas M, Seshan V, Tu S, Twaddell WS, Hegyi P, Lazar G, Steele I, et al. 2008. Global hypomethylation of genomic DNA in cancer-associated myofibroblasts. Cancer Res 68: 9900-9908.

Joesting MS, Perrin S, Elenbaas B, Fawell SE, Rubin JS, Franco OE, Hayward SW, Cunha GR, Marker PC. 2005. Identification of SFRP1 as a candidate mediator of stromal-to-epithelial signaling in prostate cancer. Cancer Res 65: $10423-10430$.

Kiskowski MA, Jackson RS II, Banerjee J, Li X, Kang M, Iturregui JM, Franco OE, Hayward SW, Bhowmick NA. 2011. Role for stromal heterogeneity in prostate tumorigenesis. Cancer Res 71: 3459-3470.

Kohonen-Corish MR, Sigglekow ND, Susanto J, Chapuis PH, Bokey EL, Dent OF, Chan C, Lin BP, Seng TJ, Laird PW, et al. 2007. Promoter methylation of the mutated in colorectal cancer gene is a frequent early event in colorectal cancer. Oncogene 26: 4435-4441.

Kretzmer H, Bernhart SH, Wang W, Haake A, Weniger MA, Bergmann AK, Betts MJ, Carrillo-de-Santa-Pau E, Doose G, Gutwein J, et al. 2015 . DNA methylome analysis in Burkitt and follicular lymphomas identifies differentially methylated regions linked to somatic mutation and transcriptional control. Nat Genet 47: 1316-1325.

Lawrence MG, Taylor RA, Toivanen R, Pedersen J, Norden S, Pook DW, Frydenberg M, Australian Prostate Cancer BioResource, Papargiris MM, Niranjan B, et al. 2013. A preclinical xenograft model of prostate cancer using human tumors. Nat Protoc 8: 836-848.
Leach DA, Panagopoulos V, Nash C, Bevan C, Thomson AA, Selth LA, Buchanan G. 2017. Cell-lineage specificity and role of AP-1 in the prostate fibroblast androgen receptor cistrome. Mol Cell Endocrinol 439: 261-272.

McLean CY, Bristor D, Hiller M, Clarke SL, Schaar BT, Lowe CB, Wenger AM, Bejerano G. 2010. GREAT improves functional interpretation of cis-regulatory regions. Nat Biotechnol 28: 495-501.

Nash C, Boufaied N, Mills IG, Franco OE, Hayward SW, Thomson AA. 2017. Genome-wide analysis of AR binding and comparison with transcript expression in primary human fetal prostate fibroblasts and cancer associated fibroblasts. Mol Cell Endocrinol. doi: 10.1016/j.mce.2017.05.006.

Niranjan B, Lawrence MG, Papargiris MM, Richards MG, Hussain S, Frydenberg M, Pedersen J, Taylor RA, Risbridger GP. 2013. Primary culture and propagation of human prostate epithelial cells. Methods Mol Biol 945: 365-382.

Olumi AF, Grossfeld GD, Hayward SW, Carroll PR, Tlsty TD, Cunha GR. 1999. Carcinoma-associated fibroblasts direct tumor progression of initiated human prostatic epithelium. Cancer Res 59: 5002-5011.

Orr B, Riddick AC, Stewart GD, Anderson RA, Franco OE, Hayward SW, Thomson AA. 2012. Identification of stromally expressed molecules in the prostate by tag-profiling of cancer-associated fibroblasts, normal fibroblasts and fetal prostate. Oncogene 31: 1130-1142.

Paland N, Kamer I, Kogan-Sakin I, Madar S, Goldfinger N, Rotter V. 2009. Differential influence of normal and cancer-associated fibroblasts on the growth of human epithelial cells in an in vitro cocultivation model of prostate cancer. Mol Cancer Res 7: 1212-1223.

Qiu W, Hu M, Sridhar A, Opeskin K, Fox S, Shipitsin M, Trivett M, Thompson ER, Ramakrishna M, Gorringe KL, et al. 2008. No evidence of clonal somatic genetic alterations in cancer-associated fibroblast from human breast and ovarian carcinomas. Nat Genet 40: 650-655.

Radulovich N, Leung L, Ibrahimov E, Navab R, Sakashita S, Zhu CQ Kaufman E, Lockwood WW, Thu KL, Fedyshyn Y, et al. 2015. Coiledcoil domain containing 68 (CCDC68) demonstrates a tumor-suppressive role in pancreatic ductal adenocarcinoma. Oncogene 34: 4238-4247.

Rodriguez-Canales J, Hanson JC, Tangrea MA, Erickson HS, Albert PS, Wallis BS, Richardson AM, Pinto PA, Linehan WM, Gillespie JW, et al. 2007. Identification of a unique epigenetic sub-microenvironment in prostate cancer. J Pathol 211: 410-419.

Seemayer TA, Lagacé R, Schürch W, Tremblay G. 1979. Myofibroblasts in the stroma of invasive and metastatic carcinoma: a possible host response to neoplasia. Am J Surg Pathol 3: 525-533.

Sing T, Sander O, Beerenwinkel N, Lengauer T. 2005. ROCR: visualizing classifier performance in R. Bioinformatics 21: 3940-3941.

Stadler MB, Murr R, Burger L, Ivanek R, Lienert F, Schöler A, van Nimwegen E, Wirbelauer C, Oakeley EJ, Gaidatzis D, et al. 2011. DNA-binding factors shape the mouse methylome at distal regulatory regions. Nature 480: $490-495$.

Taberlay PC, Achinger-Kawecka J, Lun AT, Buske FA, Sabir K, Gould CM, Zotenko E, Bert SA, Giles KA, Bauer DC, et al. 2016. Three-dimensional disorganization of the cancer genome occurs coincident with longrange genetic and epigenetic alterations. Genome Res 26: 719-731.

Taylor BS, Schultz N, Hieronymus H, Gopalan A, Xiao Y, Carver BS, Arora VK, Kaushik P, Cerami E, Reva B, et al. 2010. Integrative genomic profiling of human prostate cancer. Cancer Cell 18: 11-22.

Vizoso M, Puig M, Carmona FJ, Maqueda M, Velásquez A, Gómez A, Labernadie A, Lugo R, Gabasa M, Rigat-Brugarolas LG, et al. 2015. Aberrant DNA methylation in non-small cell lung cancer-associated fibroblasts. Carcinogenesis 36: 1453-1463.

Whiteland H, Spencer-Harty S, Morgan C, Kynaston H, Thomas DH, Bose P, Fenn N, Lewis P, Jenkins S, Doak SH. 2014. A role for STEAP2 in prostate cancer progression. Clin Exp Metastasis 31: 909-920.

Zhao H, Ramos CF, Brooks JD, Peehl DM. 2007. Distinctive gene expression of prostatic stromal cells cultured from diseased versus normal tissues. J Cell Physiol 210: 111-121.

Received August 14, 2017; accepted in revised form March 27, 2018. 


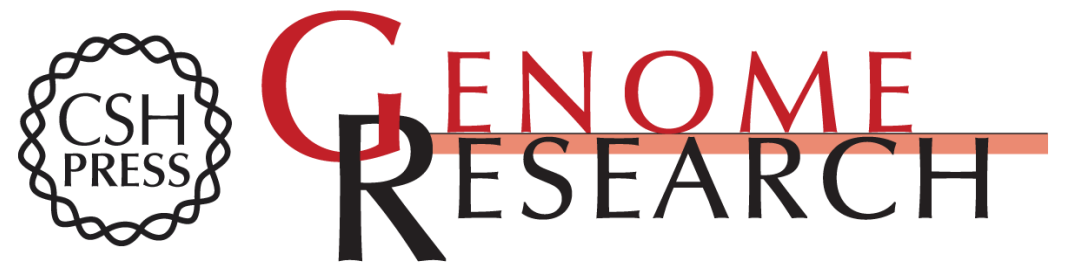

\section{Enduring epigenetic landmarks define the cancer microenvironment}

Ruth Pidsley, Mitchell G. Lawrence, Elena Zotenko, et al.

Genome Res. 2018 28: 625-638 originally published online April 12, 2018

Access the most recent version at doi:10.1101/gr.229070.117

\section{Supplemental} Material

References

Open Access

Creative Commons License

Email Alerting Service
http://genome.cshlp.org/content/suppl/2018/04/12/gr.229070.117.DC1

This article cites 51 articles, 14 of which can be accessed free at: http://genome.cshlp.org/content/28/5/625.full.html\#ref-list-1

Freely available online through the Genome Research Open Access option.

This article, published in Genome Research, is available under a Creative Commons License (Attribution-NonCommercial 4.0 International), as described at http://creativecommons.org/licenses/by-nc/4.0/.

Receive free email alerts when new articles cite this article - sign up in the box at the top right corner of the article or click here.

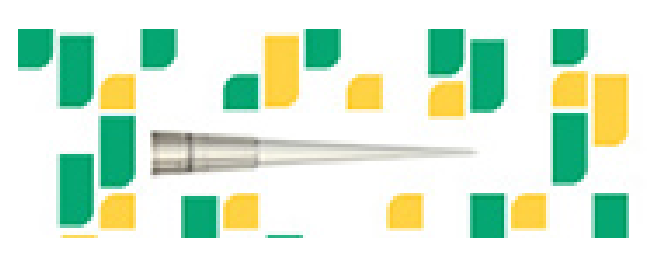

Focused on your science.

Jコగ

SCIENTIFIC

saos or seisnce

To subscribe to Genome Research go to: https://genome.cshlp.org/subscriptions 